\title{
Micromachined W-band polymeric tunable iris filter
}

\author{
Firas Sammoura $\cdot$ Liwei Lin
}

Received: 2 October 2010/ Accepted: 8 December 2010/Published online: 26 January 2011

(C) The Author(s) 2011. This article is published with open access at Springerlink.com

\begin{abstract}
A tunable filter working in the W-band spectrum has been successfully demonstrated using a combined plastic molding and electroplating process. The prototype filter architecture has two deformable membranes of $1.6 \mathrm{~mm}$ in diameter on top of a WR-10 waveguide based, two-cavity, polymeric iris filter. The membranes can be actively adjusted to deform and alter the cavity geometry concurrently for frequency tuning applications. The tunable filter was simulated using High Frequency Structure Simulator and theoretically analyzed using the perturbation method. Experimentally, a prototype band-pass filter has bandwidth of $4.05 \mathrm{GHz}$ centered at $94.79 \mathrm{GHz}$ and a minimum insertion loss of $2.37 \mathrm{~dB}$ with return loss better than $15 \mathrm{~dB}$. As a tunable filter, a total of $2.59 \mathrm{GHz}$ center frequency change has been recorded when the membranes deflected from $50 \mu \mathrm{m}$ into, to $150 \mu \mathrm{m}$ out of the waveguide. These results imply that the demonstrated tunable filter could be potentially applicable for waveguide-based mm-wave systems.
\end{abstract}

\section{Introduction}

Millimeter-wave devices have been widely used in various security and sensing applications such as weather monitoring, automobile crash avoidance, and airplane landing

F. Sammoura $(\square)$

MEMS/Sensors Division, Analog Devices Inc.,

Wilmington, MA 01887, USA

e-mail: fsammoura@hotmail.com

L. Lin

Department of Mechanical Engineering,

University of California at Berkeley,

Berkeley, CA 94720-1740, USA guidance systems (Mead et al. 1994). Tunable filters are key components in these and other systems-for example, multi-channel communication systems could use tunable filters. Various concepts and methodologies have been proposed and demonstrated for tunable filters; we briefly discuss their concepts and present the concept of polymeric tunable filter in this work.

In the general field of tunable filters, several approaches have been introduced previously, such as solid-state varactors, variable capacitors/inductors by means of microelectro-mechanical systems (MEMS) techniques (Hunter and Rhodes 1982). Typical drawbacks of CMOS varactors include high losses, unacceptable signal-to-noise (SNR) ratio, and rendered linearity. Over the past decade, radio frequency micro-electro-mechanical systems (RF MEMS) introduced an alternative way in building tunable filters for multi-band receivers. For example, MEMS varactors have been employed by (Liu et al. 2001) in order to realize a transmission line with voltage-variable electrical length. Tunable filters with $3.8 \%$ tuning range at $20 \mathrm{GHz}$ and a minimum insertion loss of $3.6 \mathrm{~dB}$ were demonstrated. Entesari and Rebeiz (2005a, b) presented wide-band tunable filters using a digital capacitor bank for $6.5-10 \mathrm{GHz}$ and $12-18 \mathrm{GHz}$ ranges with an insertion loss varying between 5.5 and $9 \mathrm{~dB}$. A reconfigurable low-pass filter was reported by (Lee et al. 2005) using multiple MEMS contact switches. The values of the inductors and capacitors were changed independently while the filter cutoff frequency dropped from 53 to $20 \mathrm{GHz}$. Robertson et al. (1995) presented a micromachined W-band bandpass filter at 94.7 GHz without tuning capability. Uher et al. (1988) described a magnetically tunable E-plane large-gap filter where the waveguide sections are symmetrically loaded with ferrite slabs. A midband tuning from about 14.1 to 15.7 GHz was achieved. 
Previously, a polymeric micromachined W-band iris filter with $3.5 \%$ bandwidth at $95 \mathrm{GHz}$ has been demonstrated (Sammoura et al. 2008b). This work presents tunable W-band, iris filters using the concept of deformable membranes to actively change the cavity sizes. Concept, design, fabrication and prototype device performances are characterized.

\section{Operation principle}

\subsection{Tunable filter design and modeling}

Figure 1a shows the schematic diagram of a 2-cavity iris filter with two deformable, circular membranes on the top surface of each cavity (Sammoura and Lin 2006). The whole device is made of plastic structure while the internal wall of the waveguide is electroplated with a $3 \mu \mathrm{m}$ thick gold layer. In the figure, $a$ and $b$ are the width and height of the rectangular waveguide, respectively; $r_{\mathrm{m}}$ is the radius of the diaphragm; $R$ is the length of the resonant cavity; $d_{1}, d_{2}$, $d_{3}$, are iris gaps and $t$ is the iris thickness. In the prototype design, the deformable diaphragm is controlled by an external pump and this could be further improved by using built-in MEMS actuators. The two-cavity resonator experiences a shift in resonant frequency upon deforming the membranes. Figure $1 \mathrm{~b}$ is a transmission line model for the tunable filter where the inductive metal planes are modeled as parallel inductive shunts of impedance, $X$, and the resonant cavities are modeled as transmission lines of electrical length $\theta_{1}$ and $\theta_{2}$, respectively (Sammoura and Lin 2007). In this 2-cavity design, $\theta_{1}$ equals $\theta_{2}$ and $X_{1}$ equals $X_{3}$ due to symmetry $\left(d_{1}=d_{3}\right)$. The deflection of membrane changes the electrical lengths of the transmission lines and the center frequency of the filter can be tuned accordingly.

The insertion loss method with binomial coefficients is used to design an iris filter with a flat passband response. A 2-cavity resonant filter is chosen such that under the same membrane deformation in both cavities, equal shift in resonant frequency of each cavity is achieved due to symmetry. For higher order filters, the membrane deflection in the cavities has to be synchronized such that filter response is preserved.

\subsection{Cavity tunability by membrane deflection}

In practical applications, resonating cavities can be tuned by either making small changes in their shape or by the insertion of small pieces of dielectric or metallic materials. In our case, changing the size of a cavity by deflecting the membranes is implemented. For small changes, perturbation techniques can be applied. Figure 2 shows a cavity perturbed by a change in shape with $V_{0}$ and $S_{0}$ are the volume and surface

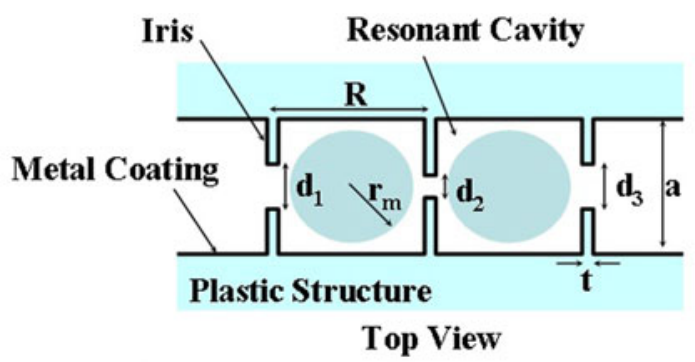

Deformable Membrane

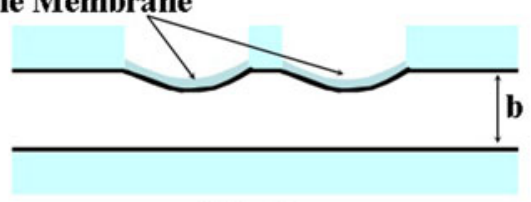

Side View

(a)

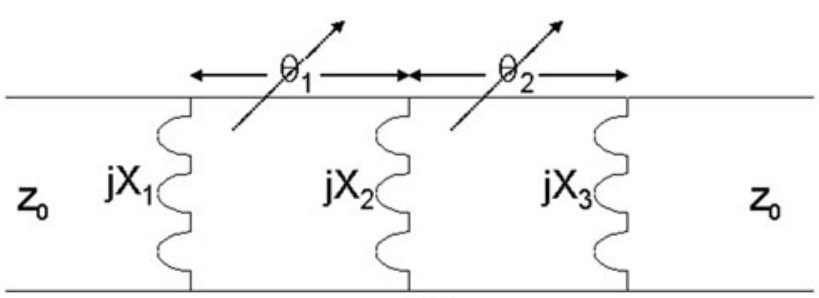

(b)

Fig. 1 a Schematic diagram of the W-band tunable filter. The resonant frequency of the cavities changes upon deforming the membranes. b Equivalent circuit of a 2-cavity iris filter where the metal layers are modeled as inductive shunts between transmission lines of variable electrical length $\theta_{1}$ and $\theta_{2}$ respectively

area of the original cavity, and $V$ and $S$ are the volume and surface area of the perturbed cavity. If $\bar{E}_{0}, \bar{H}_{0}$, and $\omega_{0}$ are the fields and the resonant frequency of the original cavity, and $\bar{E}, \bar{H}$, and $\omega$ are the fields and the resonant frequency of the perturbed cavity, then Maxwell's equations relate the change of cavity resonant frequency to the fields of the original and the tuned cavity as follows (Collin 1992):

$\omega-\omega_{o}=\frac{-j \oint_{\Delta S} \bar{E}_{0}^{*} \times \bar{H} \cdot \mathrm{d} \bar{s}}{\int_{V}\left(\varepsilon \bar{E} \cdot \bar{E}_{0}^{*}+\mu \bar{H} \cdot \bar{H}_{0}^{*}\right) \mathrm{d} v}$

where $\bar{E}_{0}^{*}$ is the conjugate of $\bar{E}_{0}$ and $\bar{H}_{0}^{*}$ is the conjugate of $\bar{H}_{0}$. This equation is an exact expression for the new resonant cavity. However, Eq. 1 is not very useful because the fields of the perturbed cavity are not generally known parameters. If we assume that $\Delta S$ is small and approximate the fields of the perturbed cavity by the fields of the original cavity, then the fractional change in resonant frequency becomes (Pozar 1997):

$\frac{\omega-\omega_{0}}{\omega_{0}} \cong \frac{\int_{\Delta V}\left(\mu\left|\bar{H}_{0}\right|^{2}-\varepsilon\left|\bar{E}_{0}\right|^{2}\right) \mathrm{d} v}{\int_{V}\left(\mu\left|\bar{H}_{0}\right|^{2}+\varepsilon\left|\bar{E}_{0}\right|^{2}\right) \mathrm{d} v}$ 
Equation (2) can be written in terms of the stored magnetic energy $W_{\mathrm{m}}$ and the stored electric energy $W_{\mathrm{e}}$ :

$\frac{\omega-\omega_{0}}{\omega_{0}}=\frac{\Delta W_{\mathrm{m}}-\Delta W_{\mathrm{e}}}{W_{\mathrm{m}}-W_{\mathrm{e}}}$

Equation 3 shows that the change in the cavity resonant frequency is a direct function of whether the cavity shape changes perturb the stored electrical energy or the stored magnetic energy. As such, the resonant frequency shift depends on both the location of the perturbation as well as whether the volume increases or decreases. When the cavity is resonating at a certain frequency, $f_{\mathrm{r}}$, the cavity looks like a real load (impedance phase equals to zero). As the center frequency shifts, the cavity will look either like an inductive or a capacitive load at $f_{\mathrm{r}}$, and hence a true phase shift is realized.

For the case of rectangular cavity operating in the $\mathrm{TE}_{101}$ mode as shown in Fig. 2, the numerator of Eq. 2 can be evaluated by integrating the fields over the sphere volume covered by the membrane. The denominator of Eq. 2 is the total energy stored in the cavity and can be calculated as (Pozar 1997):

$\int_{V_{0}}\left(\mu\left|\bar{H}_{0}\right|^{2}+\varepsilon\left|\bar{E}_{0}\right|^{2}\right) \mathrm{d} v=\frac{a b d \varepsilon A^{2}}{2}$

\subsection{Tunable iris filter simulation using HFSS}

The effect of iris thickness to the magnitudes of center frequency and bandwidth of a polymeric band-pass filter has been analyzed (Sammoura et al. 2008b) using High Frequency Structure Simulator (HFSS). As the iris

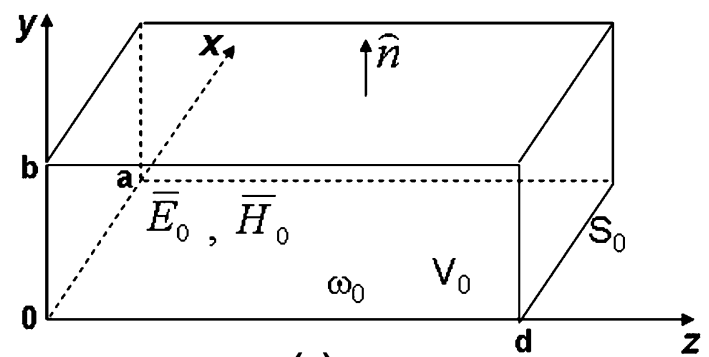

(a)

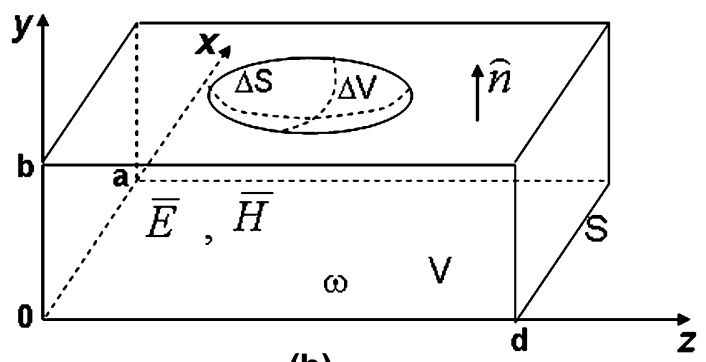

(b)

Fig. 2 A rectangular resonant cavity perturbed by membrane deflection. a a single $3 \mathrm{D}$ cavity; $\mathbf{b}$ deformed cavity thickness increases, the bandwidth decreases and the center frequency increases while the penalty is the increase of return loss. The iris thickness was set to be $300 \mu \mathrm{m}$ in this work and that is the smallest dimension that can be realized in our laboratory using precision machining to make the mold insert. The width and the height of the waveguide were 2.54 and $1.27 \mathrm{~mm}$ respectively. To realize the filter, the resonant length $R$ and the iris gaps $d_{1}$ and $d_{2}$ were calculated as $1.95,1.25$, and $0.874 \mathrm{~mm}$, respectively. Based on these values, the simulated center frequency of the prototype filter is $94.38 \mathrm{GHz}$ and bandwidth is 4.2 $\mathrm{GHz}$ with a minimum insertion loss of $0 \mathrm{~dB}$ and a return loss better than $15 \mathrm{~dB}$ over the entire band. The various parameters of the deformable membrane are also simulated using HFSS. First, it is found that the membrane diameter should be as big as possible to have large frequency tuning effects. As a result, the membrane diameter was chosen to be $1.6 \mathrm{~mm}$ to fit into the resonant cavity. Second, simulation results in Fig. 3 shows the return loss and insertion loss curves when the membrane deflected from -150 (into the waveguide) to $150 \mu \mathrm{m}$ (out of the waveguide) where the minus sign is defined as the membrane is deflected downward as shown in Fig. 1b. Table 1 summarizes the simulation results of various parameters when the membrane deflects from -150 to $150 \mu \mathrm{m}$ and a total center frequency shift of $4 \mathrm{GHz}$ is predicted, with no additional insertion loss and minimal bandwidth distortion.

The perturbation method as outlined in the previous section was used to estimate the frequency shift under varying membrane deflections. The estimated frequency shift was compared with the simulated data using HFSS as shown in Fig. 4. The predictions of the perturbation method were very close to the simulated data when membrane deflections are within $\pm 50 \mu \mathrm{m}$ where the volume change is small. For instance, at membrane deflection of $-20 \mu \mathrm{m}$, the predicted frequency shift using perturbation method is -0.29 vs $-0.25 \mathrm{GHz}$ using the HFSS simulation. However, results deviate more by these two methods under large membrane deflections.

\section{Fabrication process}

The fabrication process follows our previous work (Sammoura et al. 2006, 2008a) with the addition of the deformable membrane as illustrated in Fig. 5. The top aluminum substrate has been machined and two $1.6 \mathrm{~mm}$ in diameter holes were drilled and a $25 \mu \mathrm{m}$-thick Kapton tape with Young's Modulus of $2.5 \mathrm{GPa}$ was bonded on the aluminum substrate to form the deformable membrane in the prototype device. A seed layer of $100 \AA / 1,000 \AA \mathrm{Cr} / \mathrm{Pt}$ was sputtered on the Kapton tape as well as on the internal parts of the plastic iris filter. Following assembly with the 


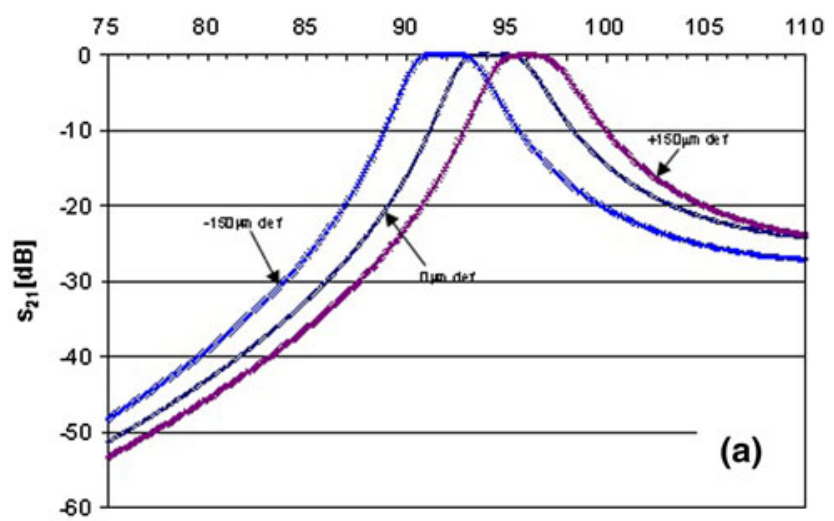

Freq [GHz]

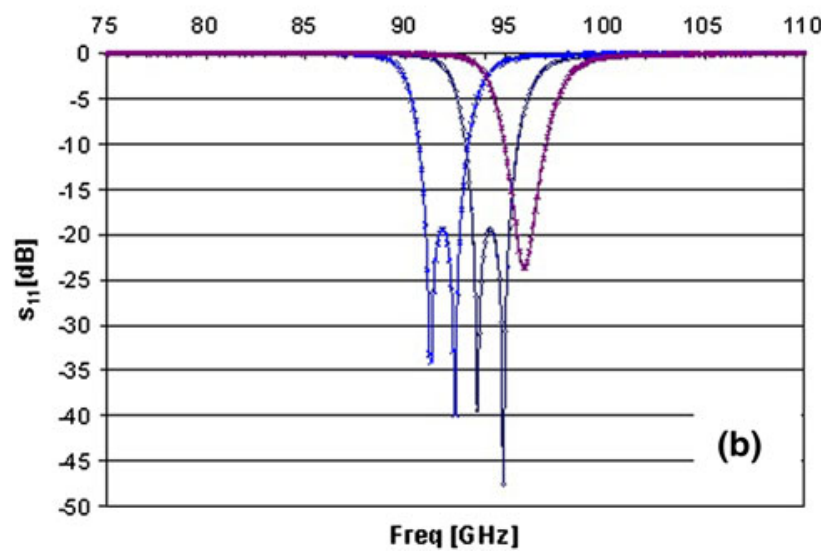

Fig. 3 Simulated a insertion loss and $\mathbf{b}$ return loss for the tunable iris filter with membrane deflection from $-150 \mu \mathrm{m}$ to $+150 \mu \mathrm{m}$

Table 1 Simulated filter parameters under various membrane deflections

\begin{tabular}{lcclcc}
\hline Deflection $(\mu \mathrm{m})$ & -150 & -50 & 0 & +50 & +150 \\
\hline$f_{\mathrm{c} 1}(\mathrm{GHz})$ & 90.00 & 91.90 & 92.30 & 93.00 & 94.15 \\
$f_{\mathrm{c} 2}(\mathrm{GHz})$ & 94.00 & 95.90 & 96.50 & 97.05 & 98.05 \\
$f_{\mathrm{c}}(\mathrm{GHz})$ & 91.98 & 93.88 & 94.38 & 95.00 & 96.08 \\
I. L $(\mathrm{dB})$ & 0.00 & 0.00 & 0.00 & 0.01 & 0.02 \\
BW $(\mathrm{GHz})$ & 4.0 & 4.0 & 4.2 & 4.05 & 3.9 \\
$\% \mathrm{BW}$ & 4.34 & 4.26 & 4.45 & 4.26 & 4.06 \\
\hline
\end{tabular}

plastic flanges, a $3 \mu \mathrm{m}$-thick gold layer was selectively electroplated to seal and provide metallization cover for the plastic, tunable iris filters. Figure 6 shows a photograph of the plastic tunable iris filter with integrated flanges, pressure tube to control the deformation of the membranes, connectors and the network analyzer.

\section{Results and discussion}

The tunable filter scattering parameters $s_{11}$ (return loss) and $s_{21}$ (insertion loss) were measured from 75 to $110 \mathrm{GHz}$

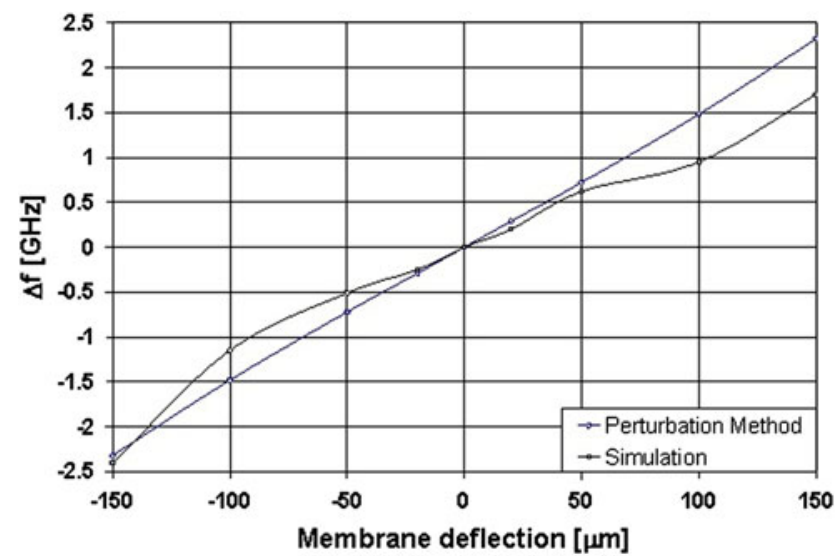

Fig. 4 Frequency shift versus membrane deflection using HFSS simulation and the perturbation method
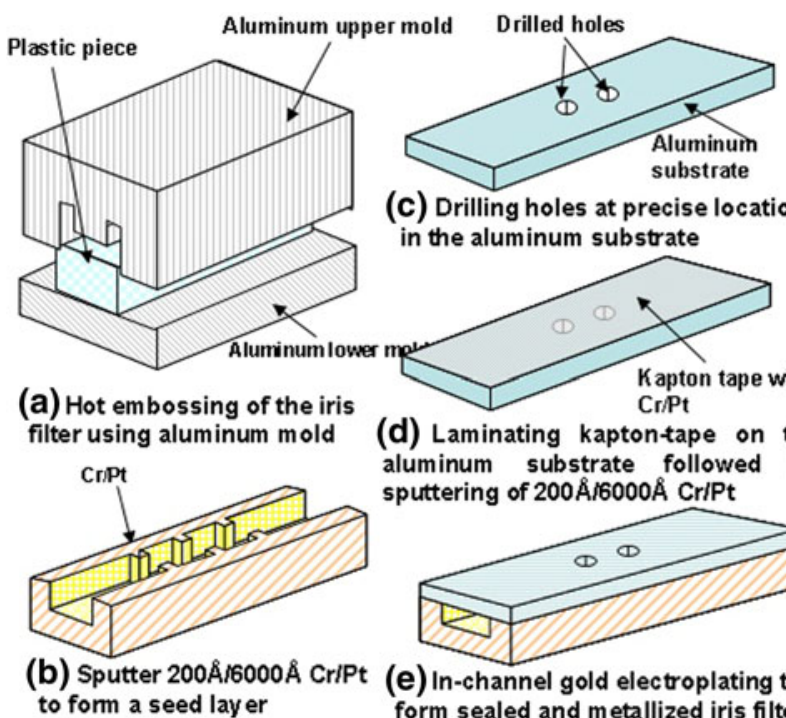

(c) Drilling holes at precise locations in the aluminum substrate

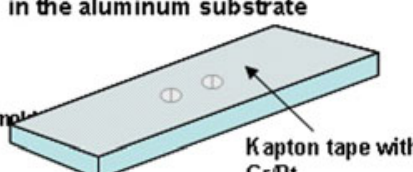

(d) Laminating kapton-tape on the aluminum substrate followed by sputtering of $200 \AA / 6000 \AA \mathrm{Cr} / \mathrm{Pt}$

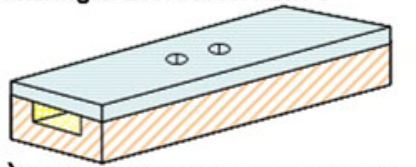

(e) In-channel gold electroplating to form sealed and metallized iris filter

Fig. 5 Fabrication process

using Anritsu ME7808B network analyzer. The membrane deflection is first characterized under a probe station. When vacuum is applied, the deflection of the membrane is about $+150 \mu \mathrm{m}$. When a pressure of $0.25 \mathrm{~atm}$ relative to the resonant cavity internal pressure is applied, membrane deflection of $-50 \mu \mathrm{m}$ is expected. These deflection data were gathered under the microscope using the focusing/ defocusing method and the accuracy can be as good as $10 \mu \mathrm{m}$. The experimental insertion loss data in Fig. 7a shows an insertion loss of $2.36,2.37$, and $2.4 \mathrm{~dB}$ when the membrane deflections are $+150,0$ and $-50 \mu \mathrm{m}$, respectively. The return loss shown is Fig. $7 \mathrm{~b}$ is always better than $15 \mathrm{~dB}$ and the center frequency drops from 96.59 to $94.79 \mathrm{GHz}$ and to $94.00 \mathrm{GHz}$. Therefore, the tuning range is $2.76 \%$ of the center frequency and Table 2 summarizes the measured tunable filter performance. The simulated data at zero deflection shows a bandwidth of $4.2 \mathrm{GHz}$ 


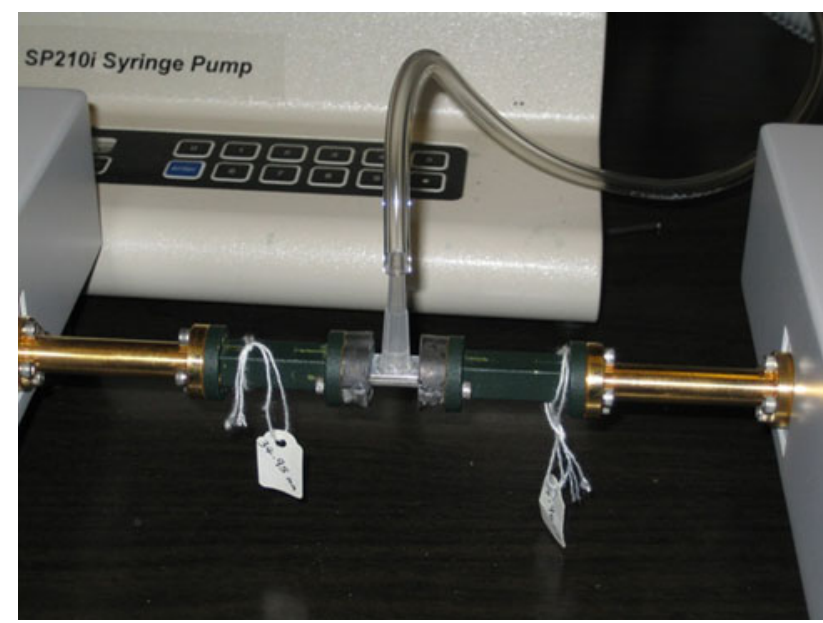

Fig. 6 Optical photo of the plastic tunable iris filter/phase shifter and experimental setup
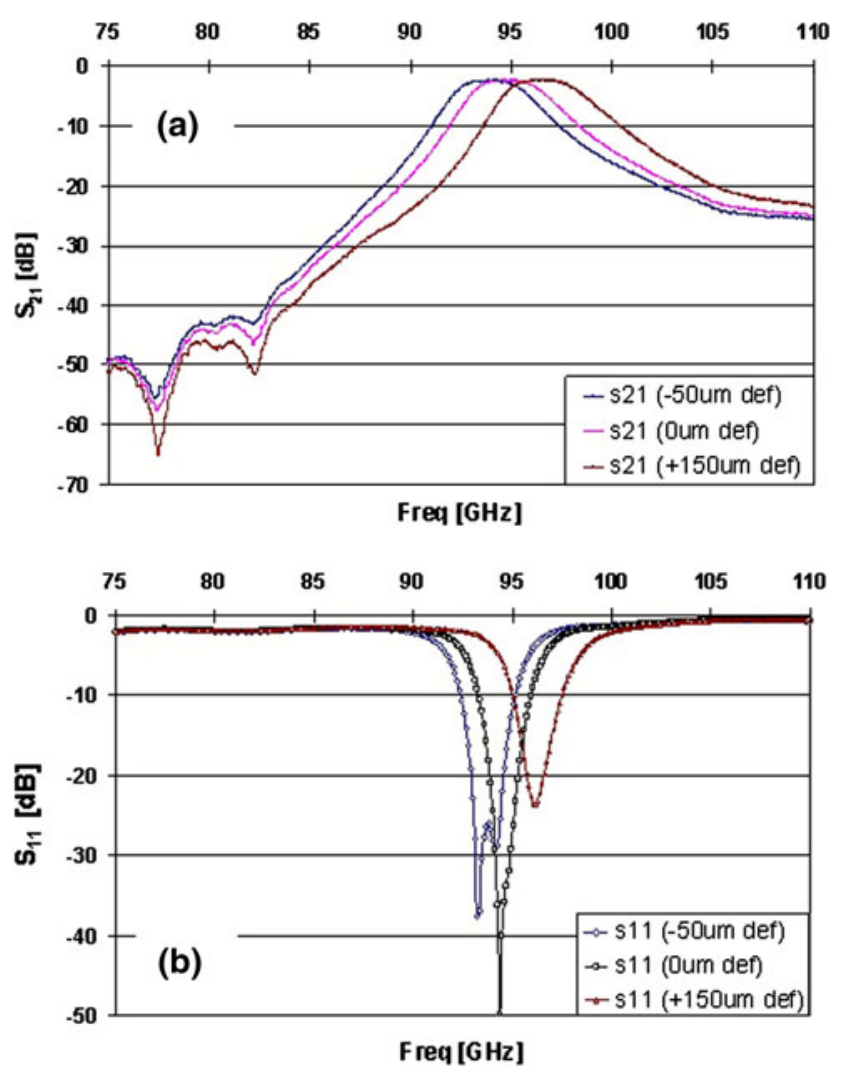

Fig. 7 Measured a insertion loss and $\mathbf{b}$ return loss of the tunable twopole filter

centered at $94.38 \mathrm{GHz}$, while the measured data shows a bandwidth of $4.05 \mathrm{GHz}$ centered at $94.79 \mathrm{GHz}$. The extra insertion loss is mainly attributed to the gap between the devices under test (DUT) and the network analyzer adaptors as observed previously from a time domain plot Sammoura et al. (2006). Figure 8 compares the measured
Table 2 Measured filter characteristics

\begin{tabular}{lccr}
\hline Deflection $(\mu \mathrm{m})$ & -50 & 0 & +150 \\
\hline$f_{\mathrm{c} 1}(\mathrm{GHz})$ & 92.00 & 92.79 & 94.48 \\
$f_{\mathrm{c} 2}(\mathrm{GHz})$ & 96.05 & 96.84 & 98.75 \\
$f_{\mathrm{c}}(\mathrm{GHz})$ & 94.00 & 94.79 & 96.59 \\
I. L $(\mathrm{dB})$ & 2.4 & 2.37 & 2.36 \\
BW $(\mathrm{GHz})$ & 4.05 & 4.05 & 4.27 \\
$\%$ BW & 4.31 & 4.27 & 4.42 \\
\hline
\end{tabular}

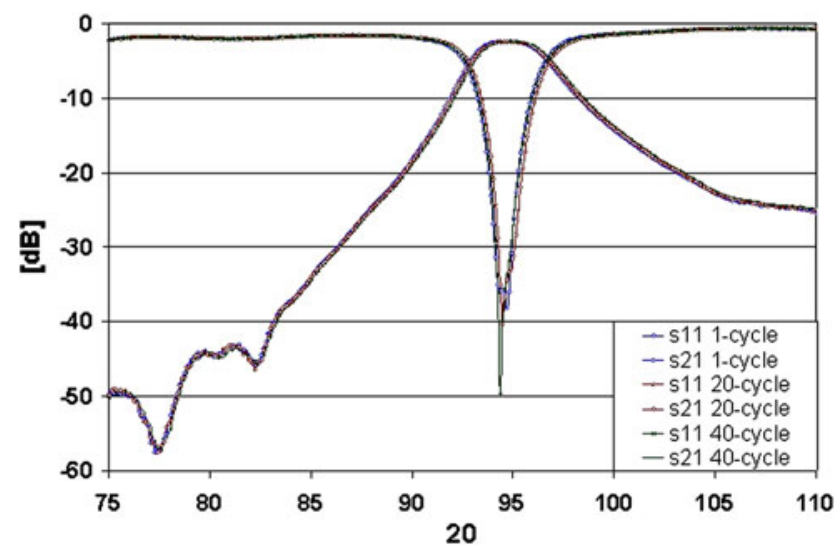

Fig. 8 Measured s-parameters for the tunable filter at zero-deflection point after 1, 20, and 40 cycles of full membrane deflection from -50 to $150 \mu \mathrm{m}$, respectively

$s$-parameters for the tunable filter at zero deflection point after one, 20, and 40 cycles of operation, respectively. It is observed that the filter response curves do not change as the membranes return to their initial positions after each cycle.

\section{Conclusion}

A plastic W-band tunable filter has been demonstrated using deformable membranes on a waveguide-based iris filter. The measured scattering parameters $s_{11}$ (return loss) and $s_{21}$ (insertion loss) data show passband of $4.05 \mathrm{GHz}$ centered at $94.79 \mathrm{GHz}$ with a minimum insertion loss of $2.37 \mathrm{~dB}$ and return loss better than $15 \mathrm{~dB}$. The center frequency can be tuned by -0.79 to $1.8 \mathrm{GHz}$ when the diaphragm is deformed between -50 and $+150 \mu \mathrm{m}$. The additional insertion loss and bandwidth distortion during the tuning operation is less than $0.11 \mathrm{~dB}$ and $0.22 \mathrm{GHz}$, respectively. A cavity perturbation method has been established and compared with HFSS simulation to characterize the device performance and both methods are consistent under small diaphragm perturbation of less than $50 \mu \mathrm{m}$. As such, this plastic, tunable W-band filter could find applications in low-cost millimeter-wave systems. 
Acknowledgments This project is supported in part by NSF grant DMI-0428884 and these devices were fabricated in the UC-Berkeley Microfabrication Laboratory and the Mechanical Engineering Machine Shop. The authors would like to thank Professor Jack Welch of UC Berkeley and J.C. Chiao of University of Texas, Arlington for valuable discussions. Mr. Elligree Bennett and Ms Wendy Penning from the machine shop of Mechanical Engineering Department at UC Berkeley helped on the mold insert fabrication and Mr. Mark Gnibus and Mr. Pat Murray from the Anritsu Corporation helped on the measurements.

Open Access This article is distributed under the terms of the Creative Commons Attribution Noncommercial License which permits any noncommercial use, distribution, and reproduction in any medium, provided the original author(s) and source are credited.

\section{References}

Collin RE (1992) Foundations of microwave engineering, 2nd edn. McGraw Hill, NewYork

Entesari K, Rebeiz G (2005a) A 12-18-GHz three-pole RF MEMS tunable filter. IEEE Trans Micro Theory Tech 53(8):2566-2571

Entesari K, Rebeiz G (2005b) A differential 4-bit 6.5-10-GHz RF MEMS tunable filter. IEEE Trans Micro Theory Tech 53(3): $1103-1110$

Hunter IC, Rhodes JD (1982) Electronically tunable microwave bandpass filters. IEEE Trans Micro Theory Tech 30(9):1354-1360

Lee S, Kim JM, Kim YK, Kwon Y (2005) Millimeter-wave MEMS tunable low pass filter with reconfigurable series inductors and capacitive switches. IEEE Microw Wirel Compon Lett 14(10): 691-693

Liu Y, Borgioli A, Nagra AS, York RA (2001) Distributed MEMS transmission lines for tunable filter applications. Int J RF Microw Comput-Aided Eng 11(5):254-260

Mead JB, Pazmany AL, Sekelsky SM, McIntosh RE (1994) Millimeter-wave radars for remotely sensing clouds and precipitation. Proc IEEE 82(12):1891-1906

Pozar DM (1997) Microwave engineering. Wiley, NewYork

Robertson S, Katehi L, and Rebeiz G (1995) Micromachined selfpackaged w-band bandpass filters. In: 1995 IEEE MTT-S international microwave symposium digest, vol 3. NewYork, pp 1543-1546

Sammoura F, Lin LW (2006) A Plastic W-band tunable iris filter. In: International microwave symposium 2006, IMS, San Francisco, pp 136-139

Sammoura F and Lin LW (2007) A Plastic W-Band MEMS Phase Shifter. The 14th International Conference on Solid-State Sensors, Actuators and Microsystems, Lyon, France, pp 647-650

Sammoura F, Su YC, Cai Y, Chi CY, Elamaran B, Lin LW, Chiao JC (2006) Plastic 95-GHz rectangular waveguides by micro molding technologies. Sens Actuators A Phys 127:270-275

Sammoura F, Fuh YK, Lin LW (2008a) Micromachined $95 \mathrm{GHz}$ waveguide-fed plastic horn antenna. J Micromech Microeng 18:055009

Sammoura F, Fuh YK, Lin LW (2008b) Micromachined plastic W-band bandpass filters. Sens Actuators A Phys 147:47-51

Uher J, Bornemann J, Arndt F (1988) Magnetically tunable rectangular waveguide E-plane integrated circuit filters. IEEE Trans Microw Theory Tech 36(6):1014-1022 